\title{
Natural Livestock Farming: Piloting a Strategy to Improve Milk Quality and Reduce Anti-Microbial Resistance
}

\author{
Katrien van't Hooft ${ }^{1 *}$, Maria Groot ${ }^{2}$ and Getachew Gebru ${ }^{3}$ \\ ${ }^{1}$ Dutch Farm Experience, and coordinator of Natural Livestock Farming network, Netherlands \\ ${ }^{2}$ Wageningen University \& Research, Netherlands \\ ${ }^{3}$ Ethiopian Society of Animal production (ESAP), Ethiopia
}

*Corresponding author: Katrien van 't Hooft, Foundation for Natural Livestock Farming, Maarsbergen, The Netherlands

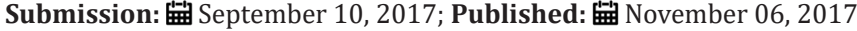

\section{Introduction}

Due to the current and expected growth of the world's population there is an increased need for high-quality animal protein. Dairy farming is regarded as one of the important ways of satisfying this need to meet the growing demand for milk, especially in developing countries. The focus on crossbreeding and increasing the productivity of dairy cattle has, besides enhanced milk production, also resulted in an increased use of agro-chemicals, mainly antibiotics and anti-parasitic drugs to keep these animals healthy. The residues of these agro-chemicals may leak into the environment, affecting natural processes, biodiversity, and soil life. Public health can also be affected due to residues in milk and meat, especially in countries with insufficient food quality controls. These processes contribute to the growing global threat of multi-resistant microbes to human and animal health [1].

\section{Natural Livestock Farming Partnership}

In 2014 a collaboration of partner organizations in India ${ }^{1}$, Ethiopia $^{2}$, Uganda $^{3}$ and the Netherlands ${ }^{4}$ was formed, looking at the possibilities to reduce the use of antibiotics and other chemicals in dairy farming, and their negative effects on public and environmental health. Organizations in the four countries joined the Foundation for Natural Livestock Farming (in short NLF-see www.naturallivestockfarming.com). Proposed activities of the network include raising consciousness about the risk of antibiotics and their effect on food quality, and implementing the five-layer approach for reducing the use of antibiotics and other chemicals.

Over the past years a joint strategy was developed, the so-called NLF 5-layered strategy (Figure 1). This integrated approach focuses on

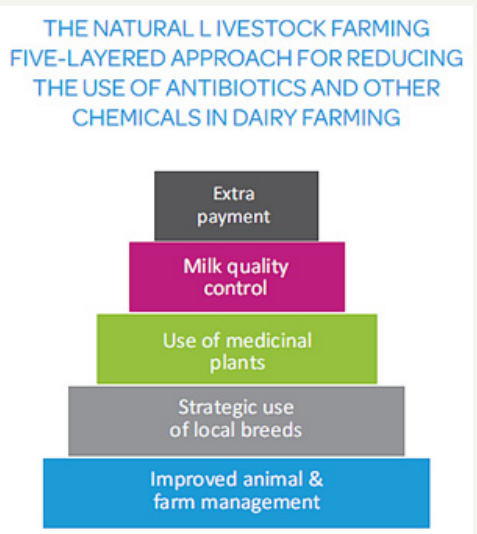

Figure 1: The Five layer strategy.

${ }^{1}$ Indian partner is Trans Disciplinary University (TDU) in Bangalore, India, collaborating with several veterinary universities and dairy companies ${ }^{2}$ Ethiopian partner is Ethiopian Society of Animal production (ESAP), in this project collaborating with Ministry of Livestock and Fisheries (MOLF) ${ }^{3}$ Ugandan partner is NLF-Uganda, in this project directly collaborating with SNV-Uganda and NARO research institute

${ }^{4}$ Netherlands partners are Dutch Farm Experience, Louis Bolk Institute and Veterinary knowledge Centre East Netherlands (VKON), in this project directly collaborating with RIKILT Wageningen Research 
I. Improving animal and farm management.

II. Genetic improvement through strategic use of local breeds.

III. Revitalizing ethno veterinary knowledge and the use of medicinal plants.

IV. Establishing quality control systems in the dairy chain.

V. Extra payment to farmers for residue-free milk.

The following five steps comprise the road map towards a system change in the dairy chain resulting in high quality residuefree milk and milk products.

\section{Piloting the NLF 5-Layered Strategy}

In the coming years we will be piloting this NLF 5-layered strategy in different projects within the four participating countries. In Ethiopia the focus is on reducing the use of antibiotics, under the applied research project entitled 'Enhancing Safety and Quality of Milk in Ethiopia' funded by NWO WOTRO [2]. In Uganda the focus is on developing alternatives for chemical tick control, to counter act the staggering resistance of ticks against acaricides. In India, the focus is on reducing antibiotic use by training farmers and veterinarians at national level on the use of herbal alternatives for the most common cattle diseases. In the Netherlands several pilot studies are being developed, including trials for the control of calf diarrhoea.

\section{Detailing the NLF Strategy}

The focus of the NLF strategy is the shift away from projects aimed at mere increase of milk production towards a more integrated focus on animal health, environmental health and quality products for human consumption (one health approach). Besides pilots, exchange visits between the different countries are another important methodology used in these projects. In this way the organizations involved learn from each other and open up to new possibilities previously unknown to them. The exchange visits are therefore essential to increase awareness about the different opportunities as well as the motivation to work towards changes at the level of farmers, veterinarians and other organizations involved.

In more technical sense, the NLF 5-layered strategy includes the following:

\section{i. Improved animal- and farm management with in ecosystem boundaries}

Improving animal health and wellbeing in dairy farming includes aspects of grass management, feeding, housing, manure management, soil fertilization and (functional) biodiversity enhancement. The Netherlands has an important experience and history on farm management to share in these areas and have developed good monitoring protocols and tools. On the topic of dairy farming within ecosystem boundaries, new initiatives and tools are emerging which can be shared with other countries. An methodological approach for participatory planning and monitoring on animal health to be used in Ethiopia and Uganda is the so-called Wheel of Animal Production and Wellbeing [3].

\section{ii. Appropriate use of local breeds in breeding strategies}

Strategic breeding for resilience to local conditions is an important component of the program. This implies strategic use of local breeds in crossbreeding programs, as well as the conservation and improvement of local breeds. Community based breeding is one of the methodologies for this, and the Ugandan and Ethiopian partners have rich experience in this area. Also within the Netherlands 'crossbreeding back' the Holstein-Frisian dairy cows with dual-purpose cattle breeds is an increasingly practiced strategy for improving cattle health and life expectancy. The improvement of local animal breeds throughout the world, often kept by remote and poor communities, represent a large and still unexplored potential in the strategy for sustainable dairy farming practices.

\section{iii. Use of medicinal plants and ethno veterinary practices:}

Medicinal plants and other natural products are a major resource for preventive, health promotion and curative purposes in dairy farming in developing countries. India has an especially rich experience and history of documenting, validating, promoting and legitimizing traditional ethno-veterinary practices in the country, and this experience is being shared across the international partners. An additional aspect is to focus on natural feeds. Natural grasslands that include herbal products are increasingly seen as an important way to reduce antibiotic use in dairy farming as well as to improve soil fertility and closing nutrient cycles. It is a small but gradually growing field in the Netherlands - and still prevalent in countries like India.

\section{iv. Milk quality control systems}

In most developing countries of the world milk quality control is limited to fat and protein content, and adulteration. Effective control of residues in fresh milk is seldom in place-while it is clear that residues in milk and milk products are increasingly common. Moreover, milk quality control is not adapted to smallholder dairy systems, currently being too expensive for small quantities of milk. At the same time the Netherlands has developed expertise in milk quality control systems, which the project aims to adapt to the conditions of smallholder dairy production.

\section{v. Extra payment for residue-free milk and sustainable enterprise development}

The $5^{\text {th }}$ step within the road map is related to farm economicsto reduce production costs and to reward dairy farmers with surplus payment for high quality and residue-free milk. This is to be combined with new market options for specialised services. For example medicinal plant enterprises will form a key component in the program for providing accessible and affordable solutions at local level while assuring equitable benefit sharing mechanisms across the value chain. 


\section{References}

1. Groot MJ, van't Hooft KE (2016) The Hidden Effects of Dairy Farming on Public and Environmental Health in the Netherlands, India, Ethiopia, and Uganda, Considering the Use of Antibiotics and Other Agro-chemicals. Front. Public Health 4:12 doi: 10.3389/fpubh.2016.00012.
2. Getachew Gebru (2017) Enhancing safety and quality of milk in Ethiopia, Applied Research Project 2017-2020 under the NWO-WOTRO scheme.

3. Katrien, van't Hooft (2016) Dairy Wheel: Successful monitoring tool for dairy farming in Bangladesh. 